\title{
Validación de un instrumento en una unidad neonatal mediante el análisis Rasch
}

\author{
Validation of an instrument in a neonatal unit through the Rasch analysis
}

Carolina Vargas-Porras' ${ }^{1}$ Beatriz Villamizar-Carvajal' ${ }^{1}$ Zayne Milena Roa-Díaz'

Para citar este artículo: Vargas-Porras C, Villamizar-Carvajal B, Roa-Díaz ZM. Validación de un instrumento en una unidad neonatal mediante el análisis Rasch. Ustasalud 2018; 17: 15-22

Licencia Creative Commons

\section{(c) (1) () $\odot$} lo tanto, los lectores pueden acceder libremente a los artículos en su formato .pdf, igualmente podrán descargarlos y difundirlos; sin embargo no podrán modificarlos o alterarlos, adicionalmente se debe reconocer la autoría de las personas que figuran en las publicaciones, pero estas no podrán ser comercializadas.

\section{RESUMEN}

Objetivo: Determinar la validez de constructo del resultado de enfermería “Desempeño del Rol de Padres" en madres de recién nacidos prematuros hospitalizados.

Materiales y métodos: Se realizó un estudio de evaluación de tecnologías diagnósticas en madres de recién nacidos prematuros hospitalizados menores o igual a 34 semanas de gestación. Se hizo análisis descriptivo y de consistencia interna desde la teoría clásica (alfa de Cronbach) y la validez de constructo del resultado de enfermería "Desempeño del rol de padres" se hizo mediante la metodología Rasch siguiendo los postulados de Messick, bajo la estructuración de Wolfe y Smith.

Resultados: Participaron 63 madres, la mediana de edad 22 años, 67\% pertenecían a los estratos socioeconómicos 1 y 2, la mediana de edad gestacional fue 33 semanas. Los 6 indicadores del resultado NOC (2211) presentaron un alfa de Cronbach $(0,901)$, valores Outfit entre 0,74 a 1,60 y explicaron el 53,8\% de la varianza; en el primer contraste la varianza no explicada fue de 1,5 autovalores, estos hallazgos señalaron que este instrumento mide el constructo del desempeño del rol de padres, es unidimensional y los datos obtenidos se ajustan al modelo Rasch.

Conclusión: El diseño, la validez y la confiabilidad de esta escala es un aporte importante a la práctica de enfermería neonatal, al tratarse del primer instrumento de fácil diligenciamiento en una unidad neonatal para evaluar el desempeño del rol materno previo egreso de las unidades de hospitalización.

Palabras clave: Estudio de validación, enfermería, recién nacido prematuro, madres.

\section{ABSTRACT}

Objective: To determine the construct validity of the nursing outcome "Performance of the Role of Parents" in mothers of hospitalized preterm infants.

Methods: An evaluation study of diagnostic technologies was conducted. The sample consisted of 63 mothers of hospitalized preterm infants, defined as $\leq 34$ weeks of gestation. A descriptive and internal consistency analysis was performed from the classical theory (Cronbach's alpha) and the construct validity of the nursing outcome "Performance of the role of parents" was evaluated using the Rasch methodology following the postulates of Messick, under the structuring of Wolfe and Smith.

Results: Sixty-three mothers were evaluated; median age 22 years, $67 \%$ belonged to socioeconomic strata 1 and 2 , the median gestational age was 33 weeks. The 6 indicators of the NOC result (2211) presented an alpha of Cronbach $(0,901)$. Outfit values between 0,74 to 1,60 and explained $53,8 \%$ of the variance; the unexplained variance in the first contrast was 1,5 eigenvalues, these findings indicate that this instrument that measures the parent role performance construct, is unidimensional and the data obtained is adjusted to the Rasch model.

Conclusion: The design, validity and reliability of this scale is an important contribution to the neonatal nursing practice, since it is the first easy-to-use instrument in the neonatal unit to evaluate the performance of the maternal role, before discharge.

Keywords: Validation study, nursing, premature newborn, mothers.
1 Grupo de investigación GRINFER, Escuela de Enfermería, Facultad de Salud, Universidad Industrial de Santander.

Autor de correspondencia:

Carolina Vargas Porras.

Correo electrónico:

carvarpo@uis.edu.co

Recibido para publicación:

11 de septiembre 2017

Aceptado para publicación

27 de agosto 2018 


\section{INTRODUCCIÓN}

El nacimiento de un recién nacido pretérmino (RNPT) es considerado como uno de los eventos más estresantes para los progenitores, puede interferir en la habilidad para la realización de las funciones necesarias e inherentes a su rol e incrementar la sintomatología de angustia emocional o física en uno o más miembros de la familia ${ }^{1}$.

Durante la hospitalización, el apoyo a los padres se centra en la explicación de la situación clínica, el tratamiento, posibles complicaciones del RNPT y tramitología administrativa, y es solo al momento del egreso cuando se le da educación y preparación a la madre sobre aspectos relacionados con el desempeño del rol materno como el baño, el masaje, la higiene, el cuidado del muñón umbilical y el manejo de los medicamentos; pero en ese corto momento la madre no logra aprovechar toda la información suministrada ${ }^{2}$.

De acuerdo con lo anterior, el período inmediato después del egreso de una Unidad de Cuidados Intensivos Neonatales (UCIN), es una situación crítica para los padres, porque ellos se mueven desde el ambiente hospitalario considerado como "seguro" hacia la casa, en donde deben asumir todas las responsabilidades y actividades del cuidado de su hijo. Es un período en el cual, pueden suceder diversos eventos adversos, si los padres durante la estancia hospitalaria de su hijo no lograron el conocimiento necesario y las habilidades para el cuidado del RNPT en casa ${ }^{3}$.

La literatura muestra que el RNPT puede requerir entre 20 a 29 visitas ambulatorias al pediatra durante el primer año y hasta un $74 \%$ más que un recién nacido a término (RNT); encontrándose que las principales razones para la readmisión y la consulta a las salas de urgencias pediátricas, no están necesariamente relacionadas con el nacimiento prematuro per se y aún no se conoce de forma precisa cuántos de estos problemas son causados por inadecuada parentalidad y cuidado en casa ${ }^{4}$.

Al considerar que la hospitalización del RNPT genera estrés materno y dificultad en la interacción madre e hijo y en este contexto, las madres se enfrentan a retos en el desempeño del rol materno ${ }^{5,6}$, es necesario que la enfermera neonatal durante el tiempo de hospitalización del recién nacido, no solo brinde cuidado a las necesidades fisiológicas del recién nacido, sino que también brinde apoyo a las necesidades maternas que influyen directamente en la evolución del estado de salud del bebé y pueden facilitar el desempeño del rol materno ${ }^{7}$. Para ello se necesita un instrumento válido que evalúe el desempeño del rol materno durante la hospitalización del RNPT; que sea práctico y de fácil diligenciamiento para la intervención por parte de enfermería en las unidades neonatales.

Enfermería cuenta con la clasificación de resultados - Nursing Outcomes Clasification (NOC), desarrollada por investigadores de la Universidad de Iowa, la cual es objeto de constante evaluación con el fin de determinar la validez y la confiabilidad en varios escenarios de aplicación y en las últimas décadas esta clasificación ha sido enfocada en la determinación de resultados en respuesta a las acciones de salud ${ }^{8}$. Estos resultados de enfermería están organizados en indicadores medibles con escalas tipo Likert, que deben cuantificarse antes y después de las intervenciones de enfermería, a fin de constatar la evolución de los pacientes. Cada resultado refleja, a través de sus indicadores, los estados o percepciones de los individuos, enmarcándose en un sistema lógico y estructurado conceptualmente, con un componente numérico que permite ampliar el estudio y el tratamiento analítico de la información. Diversos autores ya han usado con éxito estos indicadores para construir herramientas clinimétricas en distintos campos ${ }^{9,10}$.

Se han construido instrumentos a partir de algunas etiquetas del NOC para evaluar la eficacia y el efecto de intervenciones de enfermería y se ha realizado su validez; estos estudios señalan la importancia de continuar trabajando con los resultados de enfermería ${ }^{11-16}$.

La validación de resultados de enfermería se ha realizado también con el modelo de Fehring, pero desde el año 1960 el matemático danés Georg Rasch propuso un modelo de medida que permite responder a limitaciones de dicha propuesta ${ }^{17}$ y construir pruebas que brinden más información sobre el comportamiento del ítem y sujetos evaluados, permitiendo evaluar la invarianza de las medidas obtenidas. Existen estudios ${ }^{18}$ que han usado la metodología 
Rasch, en especial en el área de psicología ${ }^{19,20}$ y recientemente enfermería la ha utilizado en la validación de diagnósticos de enfermería ${ }^{21-24} \mathrm{y}$ en la validación de etiquetas de resultado ${ }^{25}$.

Al tomar en cuenta lo anterior, el objetivo del presente estudio fue determinar la validez de constructo del resultado de enfermería "Desempeño del Rol de Padres" en madres de recién nacidos prematuros hospitalizados. Este resultado es definido como las "acciones parentales para proporcionar un ambiente social, emocional y físico que alimente y sea constructivo para el niño"26.

\section{MATERIALES Y MÉTODOS}

Se realizó un estudio de evaluación de tecnologías diagnósticas en una muestra de 63 madres de recién nacidos prematuros hospitalizados en una institución de salud de tercer nivel. La muestra correspondió al tamaño sugerido por Linacre para la calibración de ítems politómicos y personas con una estabilidad de \pm 1 logit y una confianza del 99\%. Se hizo una asignación generando una secuencia de números aleatorios utilizando la función Stat-Calc del paquete estadístico Epi InfoTM 6,02.

Los criterios de inclusión fueron: ser madre por primera vez de un único prematuro hospitalizado menor o igual a 34 semanas de gestación. Los criterios de exclusión: ser madres con alteraciones psiquiátricas, dificultades en el lenguaje o madres de recién nacidos con malformaciones.

La etiqueta de resultado "Desempeño del rol de padres" cuenta con 32 indicadores, de los cuales basados en la literatura y el criterio de expertos, se eligieron diez para el presente estudio; estos se operacionalizaron a través de ítems en una 'lista de chequeo' que fue sometida a validez facial con las enfermeras de la unidad neonatal, quienes además verificaron que los ítems propuestos fueran susceptibles de observación en las madres durante su visita en la unidad neonatal, tras lo cual se decidió eliminar cuatro indicadores de la etiqueta de resultado.

Finalmente, bajo el criterio de objetividad en la evaluación por parte del personal de enfermería y temporalidad (estancia en unidad neonatal), se realizó la operacionalización de seis indicadores a través de 18 ítems; de esta forma, cada indicador contó con tres categorías de respuesta que calificaban el desempeño del rol de madre, de acuerdo con el cumplimiento de los ítems propuestos en la lista de chequeo, el puntaje total se obtuvo promediando los puntajes de los seis indicadores.

En el lapso de un año (2015) una enfermera de la UCIN previamente entrenada se encargó de la obtención del consentimiento informado y la aplicación de la lista de chequeo del estudio a cada madre que se encontraba al lado de la incubadora durante el horario de visitas en la unidad.

Se hizo doble digitación y validación de datos en Epidata 3.1; el análisis descriptivo y de consistencia interna en Stata $12.0^{27}$; la validez de constructo del resultado de enfermería "Desempeño del Rol de Padres" se realizó mediante la metodología Rasch, en el programa estadístico Winsteps ${ }^{28}$.

Se calculó el alfa de Cronbach y se realizó un análisis de validez de constructo siguiendo los postulados de Messick ${ }^{29}$, bajo la estructuración de Wolfe y $\mathrm{Smith}^{30}$. Se evaluaron algunos de los aspectos mencionados por Messick como lo son la validez sustantiva en donde se esperó que el valor del estadístico denominado coherencia (medidas que implican categorías y categorías que implican medidas) superara el 40\%, el Outlier-Sensitive Fit (Outfit) estandarizado (ZSTD) de las personas se mantuviese entre \pm 3 . La validez de contenido se evaluó a través de los Outfit and Infit Mean Square (MNSQ) cuyos valores se deben presentar en un rango entre 0,6 y 1,4 , aquí se destaca que el valor ideal para estos estadísticos es 1 . La correlación entre indicadores y medidas se espera que presente cualquier valor positivo. La validez estructural es un aspecto que exploró la unidimensionalidad a través del análisis de componentes principales de los residuales del modelo Rasch, en donde no se debían encontrar patrones; la varianza explicada por los indicadores y personas evaluadas en el modelo debió superar el $50 \%$ y en el primer contraste no podía superar el 5\% o 3 autovalores. Por último, se realizó el mapa de Wright Persona - Ítem. 


\section{Aspectos éticos y legales}

Se siguieron los lineamientos de la Resolución 008430 de 1993 del Ministerio de Salud de Colombia en la cual se establecen las normas para la investigación en salud. Según dicha Resolución esta investigación se clasificó como de bajo riesgo. Se tuvieron en cuenta las pautas éticas internacionales para la investigación en seres humanos de la declaración de Helsinki y el Council for International Organizations of Medical Sciences (CIOMS). Se realizó el consentimiento informado y en el caso de las madres menores de edad se firmó el asentimiento informado y el consentimiento por parte de su representante legal en forma verbal y escrita. Los investigadores estaban certificados en buenas prácticas para investigaciones en seres humanos. Se obtuvo el correspondiente aval ético institucional.

\section{RESULTADOS}

El resultado de enfermería "Desempeño del Rol de Padres" cuenta con 31 indicadores, como se expuso anteriormente, pero para este estudio se escogieron operativamente seis indicadores y se diseñaron sus respectivos ítems para evaluar como lo muestra la Tabla 1.

Tabla 1. Operacionalización del resultado NOC: "Desempeño del rol de padres" (2211)

\begin{tabular}{|c|c|c|c|c|}
\hline Indicador & 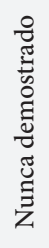 & 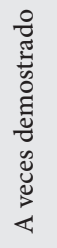 & 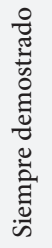 & $\begin{array}{c}\text { Operacionalización } \\
\text { Îtems }\end{array}$ \\
\hline & 1 & 2 & 3 & \\
\hline $\begin{array}{l}\text { Facilita las necesidades } \\
\text { especiales del niño. }\end{array}$ & & & & $\begin{array}{l}\text { La madre durante la visita a la unidad neonatal procura que el personal de } \\
\text { salud facilite las necesidades físicas del RN: } \\
\text { Relacionadas con las buenas condiciones higiénicas (cambio de pañal). } \\
\text { Que se encuentre confortable (abrigo). } \\
\text { Que reciba nutrición durante su ausencia. }\end{array}$ \\
\hline $\begin{array}{l}\text { Proporciona nutrición } \\
\text { adecuada a la edad. }\end{array}$ & & & & $\begin{array}{l}\text { La madre acude a la visita en la unidad neonatal y se preocupa por: } \\
\text { Aprender sobre lactancia materna. } \\
\text { Realizar extracción de la leche materna (para hacer recolección en el lactario). } \\
\text { Proporcionar alimentación por sonda o biberón o lactancia materna. }\end{array}$ \\
\hline $\begin{array}{l}\text { Interacciona de forma } \\
\text { positiva y afectiva con el } \\
\text { niño. }\end{array}$ & & & & $\begin{array}{l}\text { La madre cuando acude a la visita establecida en la unidad neonatal, interac- } \\
\text { ciona con el RN: } \\
\text { Con la voz. } \\
\text { Con la mirada. } \\
\text { Con el contacto físico (besos, caricias, etc.) }\end{array}$ \\
\hline $\begin{array}{l}\text { Demuestra empatía hacia } \\
\text { el niño. }\end{array}$ & & & & $\begin{array}{l}\text { La madre cuando acude a la visita establecida en la unidad neonatal demuestra } \\
\text { interés por: } \\
\text { Comprender las señales del RN. } \\
\text { Mostrar interés por las necesidades del RN. } \\
\text { Responder adecuadamente a las necesidades del RN. }\end{array}$ \\
\hline $\begin{array}{l}\text { Mantiene una comunica- } \\
\text { ción abierta. }\end{array}$ & & & & $\begin{array}{l}\text { La madre cuando acude a la visita señalada en la unidad neonatal: } \\
\text { Establece una comunicación con el personal de salud. } \\
\text { Muestra interés por cumplir las normas de la unidad. } \\
\text { Muestra interés por la evolución de salud del RN. }\end{array}$ \\
\hline $\begin{array}{l}\text { Expresa satisfacción con } \\
\text { el rol de madre. }\end{array}$ & & & & $\begin{array}{l}\text { La madre expresa satisfacción con el rol de madre: } \\
\text { Actitud de confianza ante el rol de madre. } \\
\text { Asume responsabilidades en su rol de madre. } \\
\text { Es optimista ante la situación del recién nacido. }\end{array}$ \\
\hline
\end{tabular}




\section{Descripción de la muestra}

Participaron 63 madres con edades comprendidas entre los 15 y 46 años, siendo la mediana de 22 años, rango intercuartílico [RIC] de 21 años; la mediana de edad gestacional fue 33 semanas [RIC] 2 semanas, $75 \%$ reportó convivir con su pareja y $67 \%$ pertenecía a los estratos socioeconómicos 1 y 2 .

\section{Validación del resultado "Desempeño del Rol de Padres"}

El coeficiente de consistencia interna, alfa de Cronbach obtuvo un valor de 0,901 para los seis indicadores, considerándose de alta confiabilidad. Se continuó con la evaluación de la validez de constructo del resultado "Desempeño del Rol de Padres" y la linealidad de la escala de medición del constructo, mediante las probabilidades de respuesta al indicador, en función de su dificultad y la habilidad de las madres ${ }^{31}$; encontrándose valores de confiabilidad de 0,78 y 0,55 y separación de 1,9 y 1,1 para personas e indicadores, respectivamente.

Por otra parte, las categorías de respuesta presentaron una eficiente diferenciación en los niveles de calificación como se observa en la Figura 1. Este funcionamiento se evidenció en todos los indicadores; la coherencia superó el valor de $40 \%$ en todas las categorías, al analizar el ajuste de las personas se encontró que la persona número 7 presentó un valor de Outfit ZSTD de 3,2 por lo tanto se eliminó, tras lo cual, se mejoraron los estadísticos observándose valores de confiabilidad de 0,80 y 0,65 y en la separación de 2,01 y 1,38 para personas e indicadores, respectivamente; justificando así continuar el análisis sin esta persona.

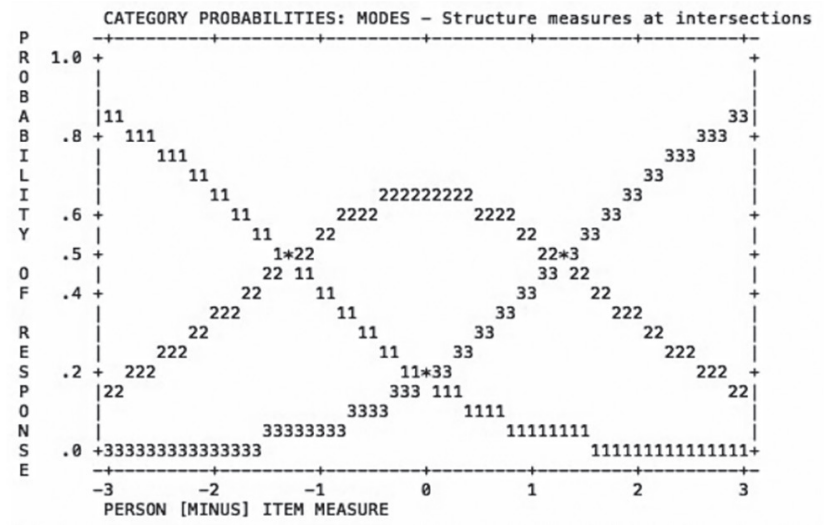

Figura 1. Curva de probabilidades de las categorías de respuesta del resultado NOC: Desempeño del Rol de Padres (2211).

Continuando con el análisis se observó que los valores de ajuste (MNSQ) Outfit se mantuvieron en el rango entre 0,74 a 1,60 (Tabla 2), todas las correlaciones indicadores - medidas presentaron valores positivos superiores a 0,70. En cuanto a la varianza, las medidas del modelo explican el $53,8 \%$ y el autovalor del primer contraste fue de 1,5; de acuerdo con todo lo mencionado, se puede concluir que los seis indicadores seleccionados para medir el constructo de "Desempeño del Rol de Padres" conforman una prueba unidimensional y que los datos obtenidos se ajustan al modelo Rasch.

Tabla 2. Dificultad, infit y outfit de los seis indicadores del resultado NOC: desempeño del rol de padres (2211)

\begin{tabular}{lccccc}
\hline \multicolumn{1}{c}{ Indicador } & Medida & \multicolumn{2}{c}{ Infit } & \multicolumn{2}{c}{ Outfit } \\
& & MNSQ & ZSTD & MNSQ & ZSTD \\
\hline Proporciona nutrición adecuada a la edad & $-0,14$ & 1,36 & 1,8 & 1,60 & 2,7 \\
\hline Mantiene una comunicación abierta & $-0,53$ & 0,97 & $-0,1$ & 1,00 & 0,1 \\
\hline Expresa satisfacción con el rol de madre & 0,96 & 0,98 & 0,0 & 0,97 & 0,0 \\
\hline Facilita las necesidades especiales del niño & $-0,14$ & 0,97 & $-0,1$ & 0,90 & $-0,5$ \\
\hline Interacciona de forma positiva y afectiva con el niño & 0,24 & 0,86 & $-0,7$ & 0,80 & $-1,0$ \\
\hline Demuestra empatía hacia el niño & $-0,38$ & 0,79 & $-1,2$ & 0,74 & $-1,4$ \\
\hline Promedio & 0,00 & 0,99 & $-0,1$ & 1,00 & 0,0 \\
\hline Desviación Estándar & 0,49 & 0,18 & 0,9 & 0,26 & 1,2 \\
\hline
\end{tabular}

Infit = Inlier-pattern-sensitive fit statistic; Outfit = Outlier-sensitive fit statistic; Medida = Lógitos; MNSQ = Mean Square; ZSTD = Standarized . 
Dados los hallazgos anteriores es importante visualizar el mapa de Wright (Figura 2), para lo cual es importante tener presente qué puntajes positivos indican mejor desempeño del rol de padres y puntajes negativos evidencian pobre desempeño del rol de padres, en la figura se puede comparar la habilidad de las madres y el grado de dificultad de los indicadores en lógitos; para este último, se puede observar que el mayor valor se obtuvo en el indicador "Expresa satisfacción con el rol de madre" con 0,96 lógitos, y que la mayoría de indicadores se ubicaron alrededor de un nivel medio de dificultad, de igual forma, existe ausencia de indicadores en las regiones extremas del mapa. Por su parte, las personas se ubicaron a lo largo de la escala, encontrándose mayores niveles de habilidad de las madres.

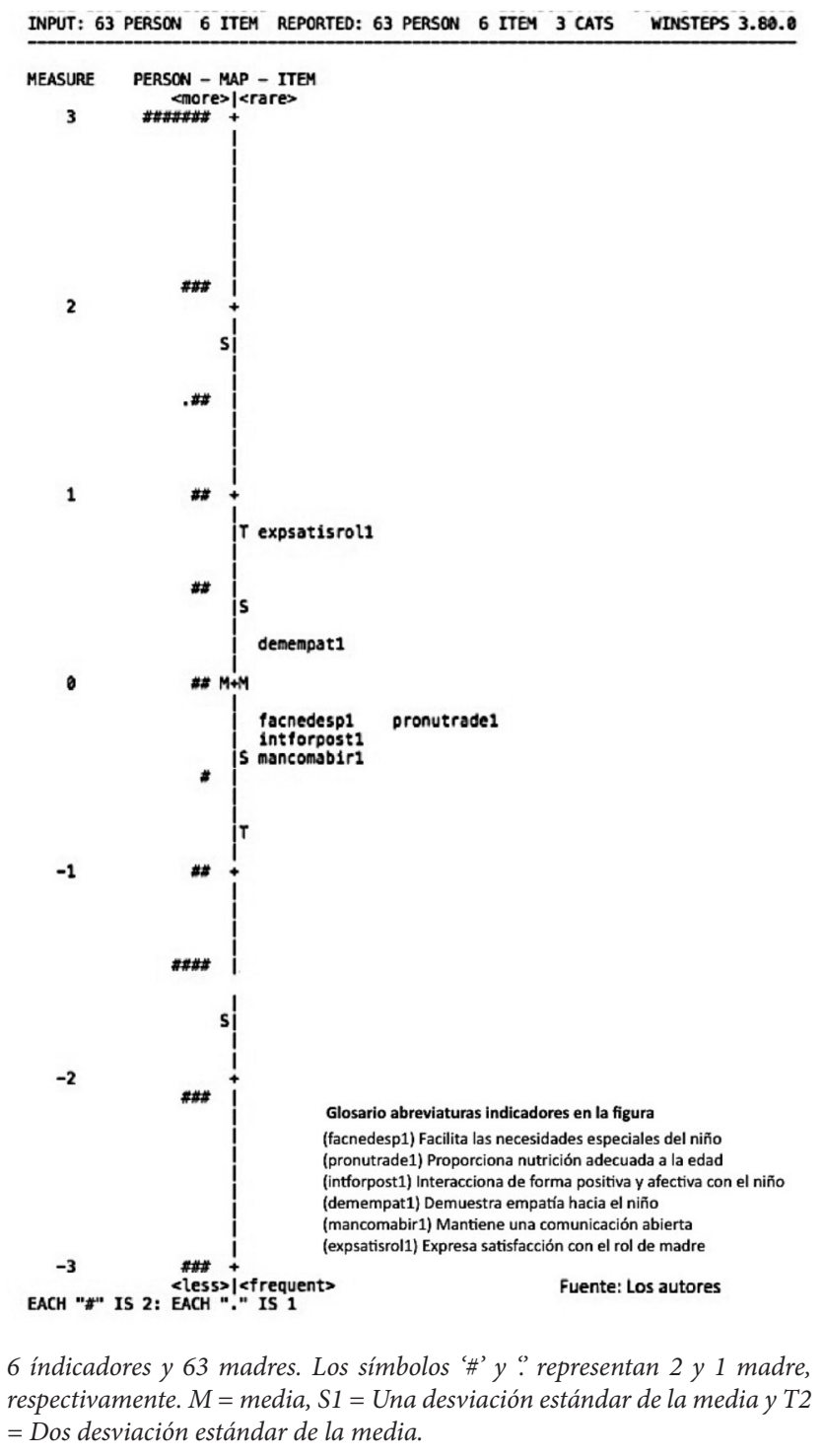

Figura 2. Mapa de Wright Persona - Ítem.

\section{DISCUSIÓN}

En general, la información presentada en este estudio soporta la validez de constructo de 5 de los 6 indicadores del resultado de enfermería "Desempeño del Rol de Padres" seleccionados para medir en madres de niños prematuros. El alto valor del alpha de Cronbach se encuentra entre los valores esperados para instrumentos ampliamente validados ${ }^{32}$. Por su parte, en los hallazgos del modelo Rasch es importante tener en cuenta que el tamaño de muestra limita la obtención de valores estables en la confiabilidad y separación, tanto de personas como indicadores, de igual forma, como se observó en el mapa de Wright, cuatro de los seis indicadores evaluados se ubicaron en niveles muy cercanos de dificultad, lo que pone en evidencia la necesidad de incluir más indicadores que logren ubicarse en los niveles desprovistos de reacti$\operatorname{vos}^{33}$; por último, se destaca la necesidad de replantear la operacionalización del indicador "Proporciona nutrición adecuada a la edad".

Son múltiples las percepciones y experiencias de la madre ante la hospitalización del RNPT, durante su estadía y al egreso. Ellas pueden experimentar temor, duelo, culpa, ansiedad, no solo durante la permanencia en la unidad de cuidado intensivo neonatal, sino también en la transición al momento del egreso, pues muchas veces; además de brindar cuidados maternos, deben ofrecer cuidados médicos debido a las complicaciones derivadas de la prematuridad. Por lo tanto, para entender e impactar mejor las causas de la readmisión de los RNPT se debe tener en cuenta el nivel del desempeño del rol materno, así como los retos que enfrentan cuando suministran el cuidado en casa ${ }^{34,35}$.

Es por esto que, el diseño, la validez y la confiabilidad de esta escala se consideran un aporte importante a la práctica de enfermería neonatal, al tratarse del primer instrumento de fácil diligenciamiento por parte del personal de enfermería para evaluar el desempeño del rol materno, que influye directamente en el cuidado del RNPT.

Una limitante de este estudio fue el número de participantes, por lo que se requiere realizar próximas investigaciones con la escala con un mayor número de muestra, incluir más indicadores, modificar la opera- 
cionalización del ítem que presentó un valor superior al rango propuesto y explorar comportamientos diferenciales. Así mismo, esta escala tuvo una aplicación intrahospitalaria y sería importante poder aplicarla en otros espacios.

Se debe tener en cuenta para próximos trabajos la inclusión de indicadores que evalúen los espectros de mayor y menor dificultad; en especial, aquellos que se distribuyan en altos niveles de dificultad, pues como se observó en el mapa de Wright, un número considerable de las participantes presentaron altos niveles del constructo evaluado.

Indicadores como "Facilita las necesidades especiales del niño" y "Proporciona nutrición adecuada a la edad" presentaron un mismo nivel de dificultad, esto se convierte en un aspecto para tener en cuenta en próximas evaluaciones de los indicadores seleccionados para este estudio, pues se puede considerar la permanencia en los instrumentos de solo uno de los dos indicadores, de acuerdo con las características de la población evaluada. Destacando que estos dos indicadores en el contexto que fueron evaluados representan gran complejidad para los padres, dado que sus hijos cursaban con una condición de prematuridad, que exige mayor complejidad en los cuidados.

Los hallazgos de este estudio, invitan a la reflexión sobre la aplicación de una escala válida para usar durante y al egreso de las unidades neonatales a las madres de RNPT, como una forma de evaluar la efectividad de las intervenciones de enfermería durante toda la estancia hospitalaria; con especial interés, en la información que se da al egreso de la unidad neonatal y su efecto en la calidad de la interacción de la madre con el RNPT, y resultados a largo plazo para estos bebés y sus familias.

\section{REFERENCIAS}

1. Howland LC. Preterm Birth: Implications for Family Stress and Coping. Newborn Infant Nurs Rev. 2007;7(1):14-19. DOI:10.1053/j.nainr.2006.12.008.

2. Delgado-Galeano M, Villamizar-Carvajal B. Coping in Mothers of Premature Newborns After Hospital Discharge. Newborn Infant Nurs Rev. 2016;16(3):105-109. DOI:10.1053/j.nainr.2016.08.007.
3. Hutchinson SW, Spillett M, Cronin M. Parents' Experiences during their Infant's Transition from Neonatal Intensive care Unit to Home: A Qualitative Study. Qual Rep. 2012;17(23):1-20.

4. Wade KC, Lorch SA, Bakewell-Sachs S, Medoff-Cooper B, Silber JH, Escobar GJ. Pediatric care for Preterm Infants after NICU discharge: High Number of Office Visits and Prescription Medications. J Perinatol. 2008;28(10):696-701.

5. Broedsgaard A, Wagner L. How to Facilitate Parents and their Premature Infant for the Transition Home. Int Nurs Rev. 2005;52(3):196-203. DOI:10.1111/j.1466-7657.2005.00414.x.

6. Kearvell H, Grant J. Getting Connected: How nurses can Support Mother/infant Attachment in the Neonatal Intensive care unit. Aust J Adv Nurs. 2010;27(3):75-82.

7. Carmona EV, Vale IND, Ohara CVDS, Abrão ACFDV. Clinical Validation of Nursing Diagnosis "Parental Role Conflict" in Mothers of Hospitalized Newborns. Int J Nurs Knowl. 2013;24(3):171-175. DOI:10.1111/j.2047-3095.2013.01251.x.

8. Seganfredo DH, Almeida, MA. Validación de contenido de resultados de enfermería según la clasificación de los resultados de enfermería (NOC) para pacientes clínicos, quirúrgicos y críticos. Rev. Latino-Am. Enfermagem. $2011 ; 9(1): 34-41$.

9. Keenan G, Stocker J, Barkauskas V, Johnson M, Maas M, Moorhead S, et al. Assessing the Reliability, Validity, and Sensitivity of Nursing Outcomes Classification in Home Care Settings. J Nurs Meas. 2003;11(2):135-155.

10. Head BJ, Aquilino ML, Johnson M, Reed D, Maas M, Moorhead S. Content Validity and Nursing Sensitivity of Community-Level Outcomes from the Nursing Outcomes Classification (NOC). J Nurs Scholarsh. 2004;36(3):251259. DOI:10.1111/j.1547-069.2004.04046.x.

11. Achury DM, Sepúlveda GJ, Rodríguez SM, Giraldo IC. Validez y confiabilidad de un instrumento evaluativo de adherencia en pacientes con falla cardíaca. Enfermería Global. 2012;11(26):1-9. DOI:10.4321/ S1695-61412012000200001.

12. Cañón W, Oróstegui M. Intervenciones educativas de enfermería en pacientes ambulatorios con falla cardíaca. Enfermería Global. 2013;12(31):52-67.

13. Sánchez S, Copé G, Gómez J, de la Rubia Montero C, Cabello J, Fernández M. Validación del NOC: Conocimiento del Régimen Terapéutico, en la identificación de 
pacientes crónicos con problemas de adaptación a sus programas de tratamiento. Enf Doc. 2007;87:10-2.

14. Aponte JC, Carrillo AJ, Lizarazo C, Silva OL. Satisfacción del cuidado de enfermería en UCI. Validez de apariencia de tres resultados del NOC. Repert. med. cir. 2012;21(2):98-107.

15. Lima JS, Lima M, Jiménez N, Domínguez I. Consistencia interna y validez de un cuestionario para medir la autopercepción del estado de salud familiar. Rev. esp. med. prev. Salud Pública. 2012;86(5):509-521.

16. Morilla-Herrera JC, Morales-Asencio JM, Fernández-Gallego MC, Berrobianco Cobos E, Delgado Romero A. Utilidad y validez de un instrumento basado en indicadores de la Nursing Outcomes Classification como ayuda al diagnóstico de pacientes crónicos de Atención Primaria con gestión ineficiente de la salud propia. An. sist. sanit. Navar. 2011;34(1):51-61.

17. Peters GJY. The Alpha and the Omega of scale Reliability and Validity: why and how to Abandon Cronbach's alpha and the Route Towards more Comprehensive Assessment of Scale Quality. European Health Psychologist. 2014;16(2):56-69.

18. Prieto G, Delgado A. Análisis de un test mediante modelo de Rasch. Psicothema. 2003;15(1):94-100.

19. Mora ZS, Prado-Calderón JE. Análisis de la validez discriminante del Eating Attitudes Test mediante el Modelo de Rasch. Reflexiones. 2015;94(1):123-135.

20. Salamanca MV, Pérez CL, Castañeda IA. Validación del cuestionario de optimismo disposicional usando la teoría de respuesta al ítem. Diversitas: Perspect. psicol. 2014;10(2):275-292.

21. Orozco LC, Villamizar B, Vargas C. Impaired Parenting in Primiparous Mothers: Clinical Validation through Rasch Analysis. Aquichan. 2015;15(2):176-187. DOI: 10.5294/ aqui.2015.15.2.2

22. Pinilla E, Orozco LC, Camargo FA, Berrío JA, Medina LX. Bullying en adolescentes escolarizados: Validación del diagnóstico de enfermería 'riesgo de violencia dirigida a otros. Hacia promoc. salud. 2012;17(1):45-58.

23. Hagquist C, Bruce M, Gustavsson JP. Using the Rasch model in Nursing Research: An Introduction and Illustrative Example. International Journal of Nursing Studies. 2009;46(3):380-393. DOI:10.1016/j.ijnurstu.2008.10.007.
24. Rodríguez-Acelas A, Cañón-Montañez W. Caminos metodológicos: validación y desarrollo de diagnósticos de enfermería. Rev Cuid. 2015;6(1):879-81. DOI: https://doi. org/10.15649/cuidarte.v6i1.248.

25. Paéz AN, Caballero LP, López N, Oróstegui M, Orozco LC, Valencia LI. Validez de constructo de un instrumento de enfermería para evaluar adherencia al tratamiento antihipertensivo. Enfermería Global. 2014;13(34):37-47.

26. Jonhson M, Maas M, Moorhead S. Clasificación de resultados de enfermería. 5a Ed. Iowa: Harcourt; 2013.

27. StataCorp. 2011. Stata Statistical Software: Release 12. College Station, TX: StataCorp LP.

28. Linacre J. Winsteps - Ministep. Rasch Model Computer Programs 3.68.0. 2009.

29. Messick S. Validity of Psychological Assessment. Validation of Inferences from Person's Responses and Performances as Scientific Inquiry into Score Meaning. American Psychologist, 1995;50(9):741-749.

30. Wolfe EW, Smith JE. Instrument Development tools and Activities for Measure Validation Using Rasch models: Part II, Validation Activities. J Appl Meas. 2007;8(2):204-234.

31. Orozco L. Medición en salud. Diagnóstico y evaluación de resultados. Un manual crítico más allá de lo básico. Bucaramanga: Publicaciones UIS; 2010.

32. Gee L, Abbott J, Conway SP, Etherington C, Webb AK. Validation of the SF-36 for the Assessment of Quality of life in Adolescents and Adults with Cystic Fibrosis. J Cyst Fibros. 2002;1(3):137-145. DOI:10.1016/ S1569-1993(02)00079-6.

33. Van der Wal MB, Tuinebreijer WE, Bloemen MC, Verhaegen PD, Middelkoop E, van Zuijlen PP. Rasch Analysis of the Patient and Observer Scar Assessment Scale (POSAS) in burn scars. Qual Life Re. 2012;21(1):13-23. DOI: 10.1007/s11136-011-9924-5.

34. Boykova M, Kenner K. Transition from Hospital to home for Parents of Preterm Infants. J. Perinat Neonat Nurs. 2012;26(1):81-87. DOI: 10.1097/JPN.0b013e318243e948.

35. Raines D. Mothers' Stressor as the day of Discharge from de NICU Approaches. Adv neonatal care. 2014;13(3):181187. DOI: 10.1097/ANC.0b013e318285fa2a. 